\title{
Pretreatment Donors after Circulatory Death with Simvastatin Alleviates Liver Ischemia Reperfusion Injury through a KLF2- Dependent Mechanism in Rat
}

\author{
Zhongzhong Liu, ${ }^{1}$ Xingjian Zhang, ${ }^{1}$ Qi Xiao, ${ }^{2}$ Shaojun Ye, ${ }^{1}$ Chin-Hui Lai, ${ }^{1}$ Jun Luo, ${ }^{1}$ \\ Xiaoying Huang, ${ }^{1}$ Wei Wang, ${ }^{1}$ Cheng Zeng, ${ }^{1}$ Zibiao Zhong, ${ }^{1}$ Xiaoli Fan, ${ }^{1}$ Zhiping Xia, \\ Yan Xiong, ${ }^{1}$ Xinfang Mao, ${ }^{3}$ Qifa Ye, ${ }^{1,2}$ and Yanfeng Wang ${ }^{1}$ \\ ${ }^{1}$ Zhongnan Hospital of Wuhan University, Institute of Hepatobiliary Diseases of Wuhan University, \\ Transplant Center of Wuhan University, Hubei Key Laboratory of Medical Technology on Transplantation, Wuhan, \\ Hubei 430071, China \\ ${ }^{2}$ Research Center of National Health Ministry on Transplantation Medicine Engineering and Technology, \\ The 3rd Xiangya Hospital of Central South University, Changsha, Hunan 410013, China \\ ${ }^{3}$ Xinjiang Key Laboratory of Biological Resources and Genetic Engineering, College of Life Science and Technology, \\ Xinjiang University, Urumqi, Xinjiang 830046, China
}

Correspondence should be addressed to Qifa Ye; yqf_china@163.com and Yanfeng Wang; yanfengwang@whu.edu.cn

Received 28 April 2017; Accepted 24 July 2017; Published 19 November 2017

Academic Editor: Hong Zheng

Copyright ( 2017 Zhongzhong Liu et al. This is an open access article distributed under the Creative Commons Attribution License, which permits unrestricted use, distribution, and reproduction in any medium, provided the original work is properly cited.

\begin{abstract}
Objective. Severe hepatic ischemia reperfusion injury (IRI) can result in poor short- and long-term graft outcome after transplantation. The way to improve the viability of livers from donors after circulatory death (DCD) is currently limited. The aim of the present study was to explore the protective effect of simvastatin on DCD livers and investigate the underlying mechanism. Methods. 24 male rats randomly received simvastatin or its vehicle. 30 min later, rat livers were exposed to warm ischemia in situ for $30 \mathrm{~min}$. Livers were removed and cold-stored in UW solution for $24 \mathrm{~h}$, subsequently reperfused for 60 min with an isolated perfused rat liver system. Liver injury was evaluated during and after warm reperfusion. Results. Pretreatment of DCD donors with simvastatin significantly decreased IRI liver enzyme release, increased bile output and ATP, and ameliorated hepatic pathological changes. Simvastatin maintained the expression of KLF2 and its protective target genes (eNOS, TM, and HO-1), reduced oxidative stress, inhibited innate immune responses and inflammation, and increased the expression of Bcl-2/ Bax to suppress hepatocyte apoptosis compared to DCD control group. Conclusion. Pretreatment of DCD donors with simvastatin improves DCD livers' functional recovery probably through a KLF2-dependent mechanism. These data suggest that simvastatin may provide a potential benefit for clinical DCD liver transplantation.
\end{abstract}

\section{Introduction}

Liver transplantation is the only successful life-saving treatment for patients with most types of end-stage liver failure [1]. However, the shortage of adequate organs all over the world has led to the use of extended-criteria donor (ECD) organs from steatotic donors or donors after circulatory death (DCD). Moreover, these DCD organs often suffer from an unpredictable longer warm ischemia time, which are much more prone to a higher risk of early allograft dysfunction (EAD) or primary graft failure (PNF) after transplantation $[2,3]$. The unavoidable hepatic ischemia reperfusion injury (IRI) induced by warm and cold ischemia is one of the main reasons of the poor outcome after liver 
transplantation $[4,5]$. Therefore, new concepts of drug and preservation have been suggested to improve graft viability and reduce hepatic IRI.

Kruppel-like factor 2 (KLF2) is a laminar flow inducible transcription factor primarily expressed by the endothelial cell and plays an important role in the regulation of endothelial function [6-8]. It induces factor expression of vasodilators and antithrombotic, antioxidant, and anti-inflammatory genes (e.g., endothelial nitric oxide synthase (eNOS), thrombomodulin (TM), and heme oxygenase-1 (HO-1)) and reduces the expression of adhesion molecules (vascular cell adhesion molecule 1 (VCAM-1) and E-selectin), conferring a vasoprotective endothelial phenotype [9-11].

Clinically, statins are antihyperlipidemic agents designed to reduce morbidity and mortality caused by cardiovascular diseases through lower cholesterol levels. In addition, statins have shown other vasculoprotective properties [12-14]. Recently, Barcelona team has demonstrated administration of simvastatin protecting the liver sinusoidal endothelium through upregulating KLF2 expression, both in experimental models of partial (70\%) hepatic warm ischemia and cold ischemia-induced acute liver injury [15-18]. Nevertheless, the potential mechanisms of DCD liver pretreatment with simvastatin remain unknown.

Therefore, in the present study, the aim was to evaluate the effects of simvastatin pretreatment on DCD livers and explore the underlying mechanisms. We hypothesized that simvastatin could improve graft viability and attenuate liver IRI via decreasing the levels of proinflammatory cytokines and reducing hepatic oxidative stress and apoptosis, which might be related to the activation of KLF2.

\section{Materials and Methods}

2.1. Experimental Design. Male Wistar rats (250-300 g) were purchased from the animal experiment center of Wuhan University. All rats were maintained on standard laboratory water and chow according to the Experimental Animal Regulations of the People's Republic of China and the Guide for the Care and Use of Laboratory Animals. All rats were fasted for $12 \mathrm{~h}$ before the experiment. The 24 rats were randomly divided into 4 groups ( $n=6$ for each group). Rats were treated with simvastatin $(1 \mathrm{mg} / \mathrm{kg}$ i.p., MedChem Express, NJ 08852, USA) or its vehicle (DMSO 0.1\%) $30 \mathrm{~min}$ [18] before in situ warm ischemia. We established a rat DCD model [19], in which through incision of the diaphragm results in cardiac arrest without prior heparinization and portal clamping. The period of in situ liver warm ischemia started from the point of cardiac arrest. In the second step, the livers procured were stored for $24 \mathrm{~h}$ at $4^{\circ} \mathrm{C}$ in University of Wisconsin (UW) solution. In the third step, we evaluated reperfusion injury using the isolated liver perfusion model.

We chose the following experimental groups:

(1) DCD simvastatin group (DCD-Sim group): simvastatin pretreatment, DCD livers exposed to $30 \mathrm{~min}$ of in situ warm ischemia followed by $24 \mathrm{~h}$ cold storage (UW solution) and subsequent $1 \mathrm{~h}$ ex vivo warm reperfusion.
(2) Cold storage reperfusion group (CSP group): normal livers without warm ischemia, subjected to $24 \mathrm{~h}$ cold storage (UW solution) and subsequent $1 \mathrm{~h}$ ex vivo warm reperfusion.

(3) DCD control group (DCD-Con group): vehicle (DMSO 0.1\%) pretreatment (no simvastatin pretreatment), DCD livers exposed to $30 \mathrm{~min}$ of in situ warm ischemia followed by $24 \mathrm{~h}$ cold storage (UW solution) and subsequent $1 \mathrm{~h}$ ex vivo warm reperfusion.

(4) DCD simvastatin group (DCD-Sim group): simvastatin pretreatment, DCD livers exposed to $30 \mathrm{~min}$ of in situ warm ischemia followed by $24 \mathrm{~h}$ cold storage (UW solution) and subsequent $1 \mathrm{~h}$ ex vivo warm reperfusion.

2.2. DCD Model and Liver Procurement. Rats were anesthetized by pentobarbital sodium $(50 \mathrm{mg} / \mathrm{kg})$ via intraperitoneal injection. After laparotomy, incision of the diaphragm resulted in cardiac arrest. Following $30 \mathrm{~min}$ warm ischemia in situ, the livers were perfused in situ with $60 \mathrm{ml} 4^{\circ} \mathrm{C}$ cold heparinized $(1 \mathrm{U} / \mathrm{ml})$ saline via the aorta abdominalis to wash out the blood. The portal vein, superior hepatic caval vein and common bile duct were cannulated. Subsequently, the infrahepatic vena caval and the right adrenal veins were ligated. Then, livers were stored at $4^{\circ} \mathrm{C}$ in UW solution for $24 \mathrm{~h}$.

2.3. The Isolated Perfused Rat Liver System (IPRL). After $24 \mathrm{~h}$ cold storage, livers were exposed $15 \mathrm{~min}$ in the chamber at room temperature to simulate a rewarming period during liver implantation. Afterwards, livers were connected to the IPRL system for 60 min normothermic reperfusion ex vivo. Livers were reperfused through the portal vein only with freshly prepared Krebs-Henseleit bicarbonate buffer saturated with $95 \% \mathrm{O}_{2}$ and $5 \% \mathrm{CO}_{2}$. Flow velocity of perfusate was controlled at a flow rate of $15 \mathrm{ml} / \mathrm{min}$ [19] by a pulsatile perfusion pump (PING Technologies Inc., Shanghai, China). Portal venous pressure was measured continuously by a BL420F pressure transducer system (Chengdu Taimeng Science and Technology Co., Chengdu, China) and adjusted to make sure that the portal venous pressure is below $8 \mathrm{mmHg}$. Samples of perfusate were collected via the superior hepatic caval vein catheter at different time points $(5,15,30$, and $60 \mathrm{~min})$. After $1 \mathrm{~h}$ warm reperfusion, samples of perfusate, liver tissue, and bile output were collected for analyses.

2.4. Transaminases of Alanine Aminotransferase (ALT), Aspartate Aminotransferase (AST), and Bile Output Analysis. Hepatocyte injury was determined by detecting ALT and AST release in perfusate (the clinical laboratory of the Central South Hospital of Wuhan University) following the manufacturer's protocols. Liver function was measured by bile output ( $\mu$ l of bile/g of liver) after ex vivo warm reperfusion.

2.5. Western Blotting Analysis. Liver samples were processed from $-80^{\circ} \mathrm{C}$ storage and Western blotting performed as described [20]. The primary antibodies used are as follows: KLF2 (1:400, rabbit anti-KLF2 antibody, Biosynthesis Biotechnology, Beijing, China), phosphorylated eNOS at 
Ser1177 (1:1000, rabbit anti-phosphorylated eNOS antibody, Cell Signaling, Danvers, MA), total eNOS (1 : 1000, rabbit anti-eNOS antibody, Cell Signaling, Danvers, MA), Bax ( $1: 1000$, rabbit anti-Bax antibody, Proteintech, Manchester, UK), and Bcl-2 (1:1000, rabbit anti-Bcl-2 antibody, Proteintech, Manchester, UK). The bands were revealed by chemiluminescence ECL reagent (Proteintech, Manchester, UK), and protein expression was quantified by densitometric analysis using the Quantity One software package (Hertfordshire, UK). All bands were assayed for $\beta$-actin ( $1: 1000$, rabbit anti- $\beta$-actin antibody, Proteintech, Manchester, UK) content as standardization of sample loading.

2.6. Malondialdehyde (MDA), Superoxide Dismutase (SOD), and Adenosine Triphosphate (ATP) Analysis. For the evaluation of oxidative stress and mitochondrial function, frozen liver tissue was homogenized with butylhydroxytolueneadded Tris- $\mathrm{HCl}$ buffer determined by using the colorimetric assay kit specific for malondialdehyde (MDA), superoxide dismutase (SOD), and ATP (Nanjing Jiancheng Bioengineering Institute, Nanjing, China) following the manufacturer's instructions. The results were measured as $\mu \mathrm{mol} /$ gprot.

2.7. Quantitative RT-PCR Analysis. Total RNA from frozen rat liver tissues was extracted using TRIzol reagent (Invitrogen Inc., Grand Island, NY, USA) and subsequent RNA was reverse-transcribed to cDNA (Thermo Scientific Revert Aid, USA) according to the manufacturer's instructions. SYBR green quantitative RT-PCR was used to assay the expression of target genes. The $\beta$-actin was detected as housekeeping gene. All primers were listed in Table 1.

2.8. Histopathology and TUNEL Staining. The paraffinfixed liver sections were stained with hematoxylin-eosin (H\&E). The liver tissue sections of IRI were graded blindly by Suzuki's criteria [21]. Histological changes were graded from 0 to 4 based on the degree of cellular vacuolization, hepatic sinusoid congestion, and hepatocyte necrosis.

Apoptosis was assayed with TUNEL staining (Roche Diagnostics, Indianapolis, IN, USA) according to the manufacturer's instructions. The total hepatocytes and TUNELpositive cells were detected in 3 random chosen views (100x) for each liver sections using a fluorescence microscope. The rate of apoptosis (number of TUNEL-positive cells/total number of hepatocytes $\times 100 \%$ ) in each view was measured with Image-Pro Plus 6.0 (Media Cybernetics, Rockville, MD, USA).

2.9. Statistical Methods. All data were analyzed using SPSS 16.0 statistical software for Windows (SPSS Inc., Chicago, IL, USA). All results are presented as the mean \pm SD. One-way ANOVA was used to compare statistical significance between groups. Statistical significance was defined as $P<0.05$.

\section{Results}

3.1. DCD Donor Pretreatment with Simvastatin Alleviates Hepatic IRI. To evaluate the effects of simvastatin pretreatment in DCD donors on hepatic injury derived from
TABLE 1: Nucleotide sequences of primers used for quantitative RT-PCR.

\begin{tabular}{lcc}
\hline Gene & & Primer sequence (5'-3) \\
\hline \multirow{2}{*}{ KLF2 } & Forward & GAGCCTATCTTGCCGTCCTT \\
& Reverse & AGCACGCTGTTTAGGTCCTC \\
\hline \multirow{2}{*}{ eNOS } & Forward & CAACTGGAAAAAGGCAGCCC \\
& Reverse & AAGAGCCTCTAGCTCCTGCT \\
\hline \multirow{2}{*}{ TM } & Forward & CCTTTGTCTTTCCGGGCTCT \\
& Reverse & TCAAGTCCTCCCTACCCTCG \\
\hline \multirow{2}{*}{ HO-1 } & Forward & CCTGCTAGCCTGGTTCAAGA \\
& Reverse & GAGTGTGAG GACCCATCGCA \\
\hline \multirow{2}{*}{ HMGB1 } & Forward & GGCGGCTGTTTTGTTGACAT \\
\hline \multirow{2}{*}{ TLR4 } & Reverse & ACCCAAAATGGGCAAAAGCA \\
\hline \multirow{2}{*}{ CD68 } & Forward & TGTATCGGTGGTCAGTGTGC \\
& Reverse & CAGCTCGTTTCTCACCCAGT \\
\hline IL-1 $\beta$ & Forward & CGTTACCCGGAGACGACAAT \\
\hline \multirow{2}{*}{ IL-6 } & Forward & TCCTTGGTGGCCTACAGAGT \\
\hline ICAM-1 & Feverse & FACTTCACCATGGAACCCGT \\
& Forward & AGAGACTTCCAGCCAGTTGC \\
& Reverse & AGTCTCCTCTCCGGACTTGT \\
\hline
\end{tabular}

ex vivo warm reperfusion, hepatic function, bile production, hepatic architecture distortion, and presence of oxidative stress, inflammation, and apoptosis were measured.

As shown in Figures 1(a) and 1(b), after donor cardiac arrest for $30 \mathrm{~min}$ in vivo and $24 \mathrm{~h}$ cold storage ex vivo, the levels of ALT and AST in the liver perfusate were remarkably increased at 5, 15, 30, and 60 min compared with those in the normal control group, while the peak was found at warm reperfusion for $60 \mathrm{~min}$. However, DCD donor pretreatment with simvastatin significantly reduced the perfusate releases of ALT and AST at each time point compared with DCD control group $(P<0.05)$. As shown in Figure $1(\mathrm{c})$, after $30 \mathrm{~min}$ warm ischemia, $24 \mathrm{~h}$ cold-stored and $1 \mathrm{~h}$ warm-reperfused, the liver grafts released a lower quantity of bile in comparison to the normal control group. The detrimental effects were remarkably improved in DCD liver pretreatment with simvastatin (Figures 1(a), 1(b), and 1(c), $P<0.05$ ).

3.2. DCD Donor Pretreatment with Simvastatin Ameliorates Hepatic Pathological Changes after Warm Reperfusion Subjected to $24 \mathrm{~h}$ Cold Preservation. Figure 2(a) shows the same trends in H\&E staining. Severe necrotic areas, cytoplasmic vacuolization of hepatocytes, and neutrophil infiltration were observed in the DCD control group, while DCD donor pretreatment with simvastatin groups showed minor injury after warm reperfusion. The results indicated that DCD donor pretreatment with simvastatin dramatically ameliorated pathological changes $(P<0.05$, Figure $2(b))$. 


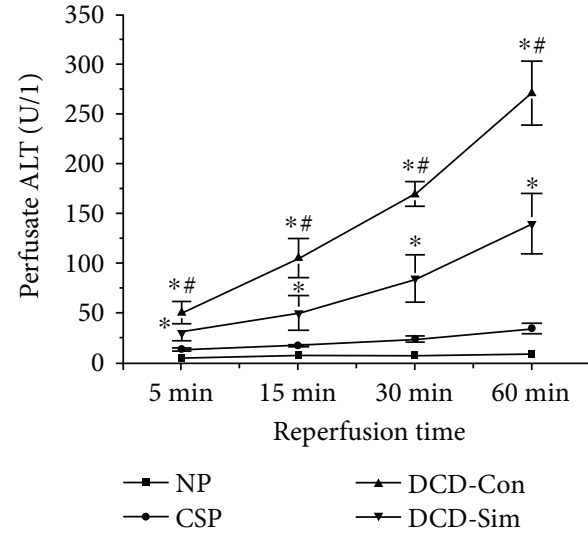

(a)

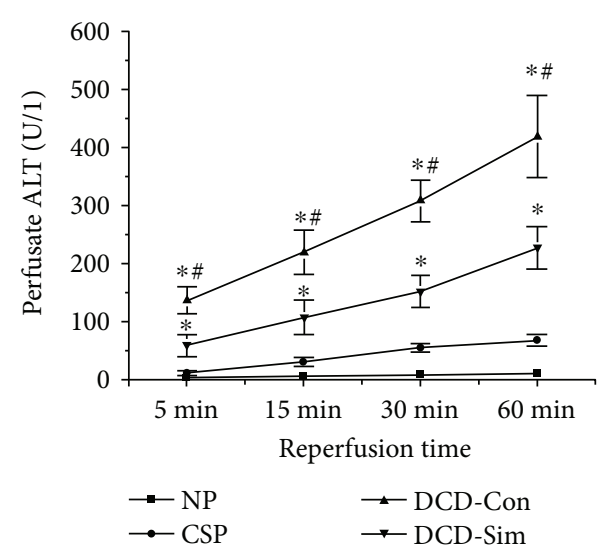

(b)

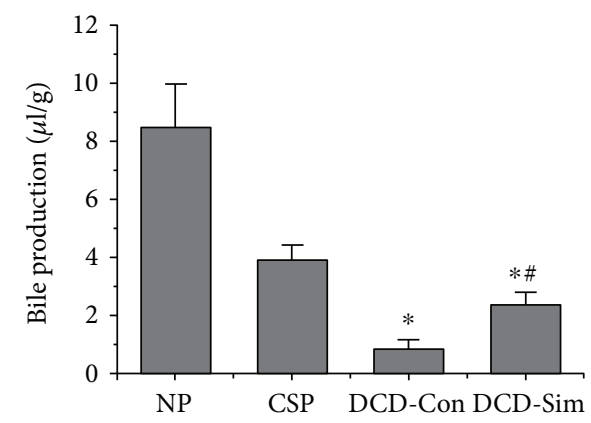

(c)

FIgURE 1: Simvastatin pretreatment alleviates hepatic IRI. Hepatic injury evaluated as release of transaminases (ALT and AST) in liver perfusate from rats at $5 \mathrm{~min}, 15 \mathrm{~min}, 30 \mathrm{~min}$, and $60 \mathrm{~min}$ time points. Perfusate ALT (a) and ALT (b) levels were expressed as the mean \pm SD ( $n=6$ per group; ${ }^{*} P<0.05$ versus NP group, ${ }^{\#} P<0.05$ versus $D C D$-Con group).

3.3. DCD Donor Pretreatment with Simvastatin Maintains the Expression of KLF2 and Its Protective Target Genes in Livers after Warm Reperfusion Subjected to $24 \mathrm{~h}$ Cold Preservation. After donor cardiac arrest for $30 \mathrm{~min}$ in vivo and $24 \mathrm{~h}$ cold storage ex vivo, the rat livers exhibited a significant reduction in KLF2 protein and mRNA expression after warm reperfusion compared with the normal control livers (Figures 3(b) and 3(e)). Meanwhile, DCD control group showed significant decreases in the expression of KLF2's protective target genes, such as eNOS (phosphorylation protein and mRNA levels, Figures 3(c) and 3(f)), TM (Figure 3(g)), and HO-1 (Figure 3(h)). DCD donor pretreatment with simvastatin effectively prevented the decay of liver KLF2, eNOS, TM, and HO- 1 after warm reperfusion subjected to $24 \mathrm{~h}$ cold preservation (Figures 3(a), 3(b), 3(c), 3(d), 3(e), 3(f), and 3 (g), $P<0.05)$.

3.4. DCD Donor Pretreatment with Simvastatin Reduces Oxidative Stress and Improves ATP Levels in Livers after Warm Reperfusion Subjected to $24 \mathrm{~h}$ Cold Preservation. As shown in Figures 4(a) and 4(b), after donor cardiac arrest for $30 \mathrm{~min}$ in vivo and $24 \mathrm{~h}$ cold storage ex vivo, the rat livers in DCD control group showed a severe increase in oxidative stress after warm reperfusion compared with the normal control livers. Moreover, DCD liver pretreatment with simvastatin significantly increased SOD and reduced MDA levels in comparison with DCD control group $(P<0.05)$.
Furthermore, as shown in Figure 4(c), DCD liver pretreatment with simvastatin significantly increased ATP levels after warm reperfusion in comparison with DCD control group, but were still significantly lower than the one in the CSP group $(P<0.05)$.

3.5. DCD Donor Pretreatment with Simvastatin Inhibits Innate Immune Responses and Inflammation in Livers after Warm Reperfusion Subjected to $24 \mathrm{~h}$ Cold Preservation. As shown in Figures 5(a), 5(b), and 5(c), DCD liver pretreatment with simvastatin significantly inhibited high-mobility group box 1 (HMGB1), Toll-like receptor 4 (TLR4), and CD68 mRNA levels after warm reperfusion in comparison with DCD control group, but were still significantly more than the normal control group $(P<0.05)$. In addition, DCD donor pretreatment with simvastatin pretreatment significantly reduced the levels of inflammatory cytokines. Realtime PCR also was used to evaluate the mRNA expression of these inflammatory factors. As shown in Figures 5(e), 5(f), and $5(\mathrm{~g})$, compared with the DCD control group, simvastatin pretreatment dramatically suppressed the mRNA expression of interleukin- $\beta$ (IL-1 $\beta$ ), IL-6, and intercellular adhesion molecule 1 (ICAM-1). These results provided strong evidence that DCD liver pretreatment with simvastatin could significantly reduce the release of HMGB1, TLR4, CD68, IL-1 $\beta$, IL-6, and ICAM-1 after warm reperfusion subjected to $24 \mathrm{~h}$ cold preservation in rats. 

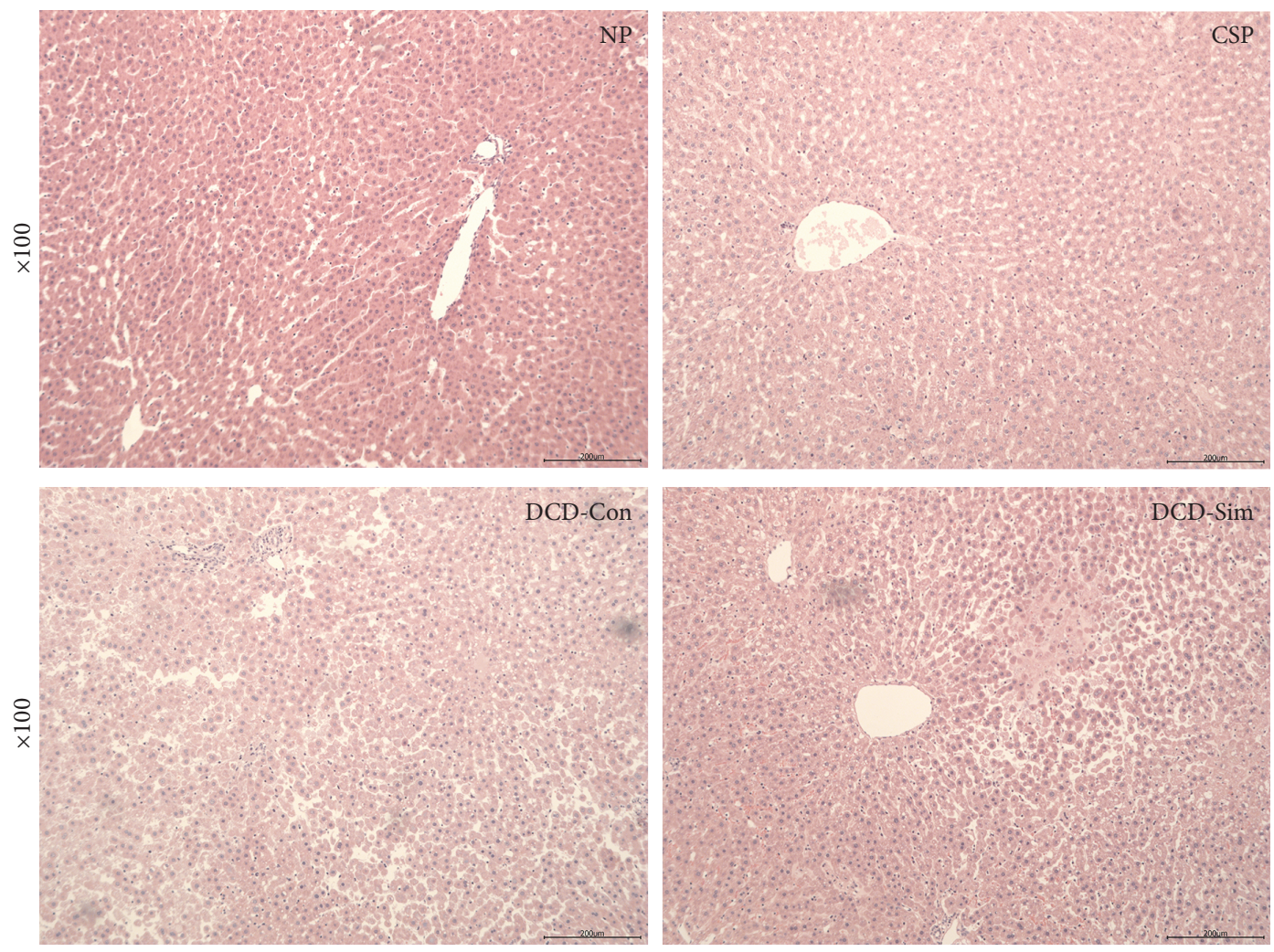

(a)

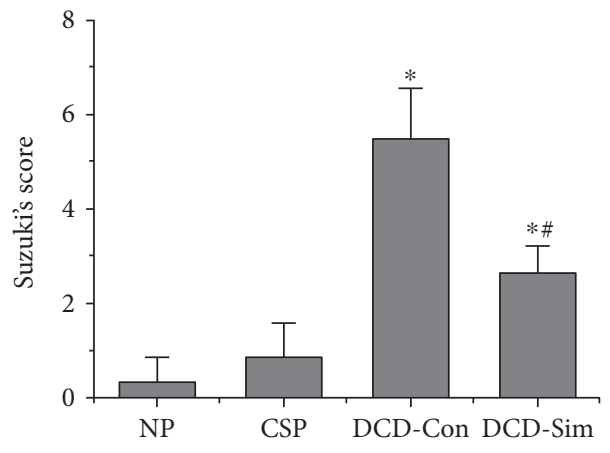

(b)

FIGURE 2: Simvastatin pretreatment ameliorates hepatic pathological changes. (a) Representative hematoxylin and eosin (HE) staining of liver tissues from rats after $1 \mathrm{~h}$ warm reperfusion. Original magnification, 100x. (b) Suzuki's histological score of liver tissue after $1 \mathrm{~h}$ warm reperfusion $\left(n=6 ;{ }^{*} P<0.05\right.$ versus NP group, ${ }^{\#} P<0.05$ versus DCD-Con group).

3.6. DCD Donor Pretreatment with Simvastatin Suppresses Hepatocyte Apoptosis in Livers after Warm Reperfusion Subjected to $24 \mathrm{~h}$ Cold Preservation. Bax and Bcl-2 are important markers of apoptosis. Bax is a proapoptotic protein, while Bcl-2 is an antiapoptotic protein. Western blotting was used to investigate the expression of markers of apoptosis at the protein level (Figures 3(a) and 3(c)). The results showed that $\mathrm{Bcl}-2 / \mathrm{Bax}$ (ratio) was downregulated in livers of the DCD control group and upregulated in DCD liver pretreatment with simvastatin group after warm reperfusion subjected to $24 \mathrm{~h}$ cold preservation $(P<0.05)$. As shown in Figure 6(a), the results of TUNEL staining demonstrated that a large amount of apoptotic cells were detected in the DCD control group and few apoptotic cells were seen in the DCD liver pretreatment with simvastatin group. It indicated statistically significant differences between the two groups by Image-Pro Plus software (Figure 6(b), $P<0.05$ ).

\section{Discussion}

Hepatic IRI injury is a complex pathological process associated with liver transplantation, shock, and trauma. The IRI is a serious threat for short- and long-term graft outcome of liver transplantation. Moreover, DCD livers have a unique process of hepatic IRI, which includes severe warm ischemia before organ procurement in vivo, more sensitive cold ischemia during the period of organ preservation in vitro, and reperfusion injury after transplantation 


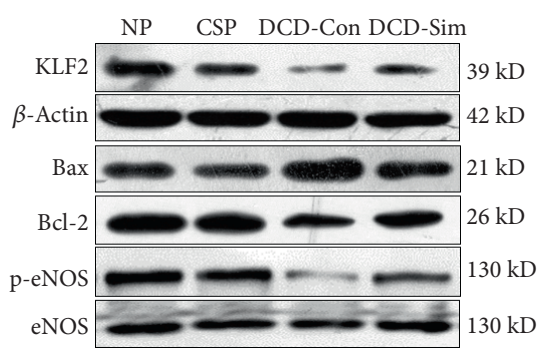

(a)

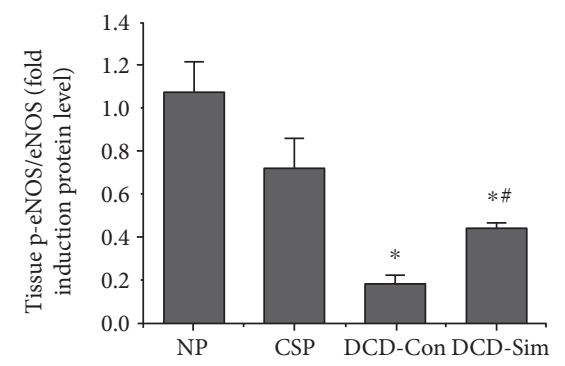

(c)

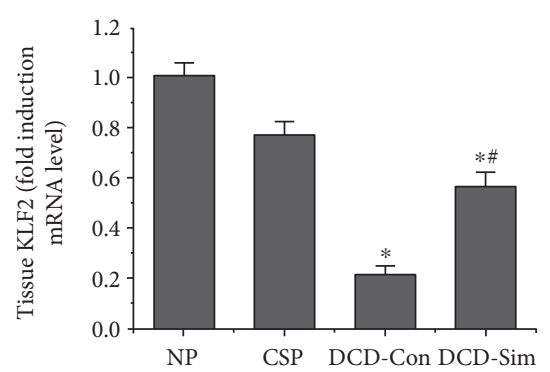

(e)

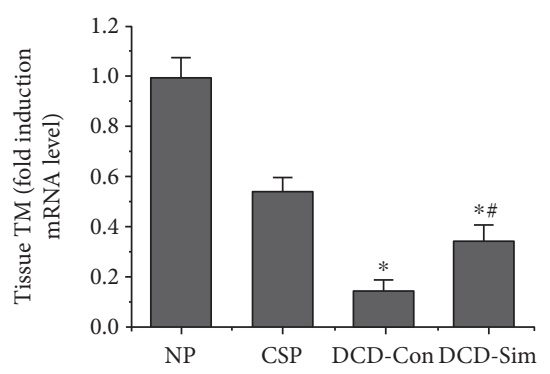

(g)

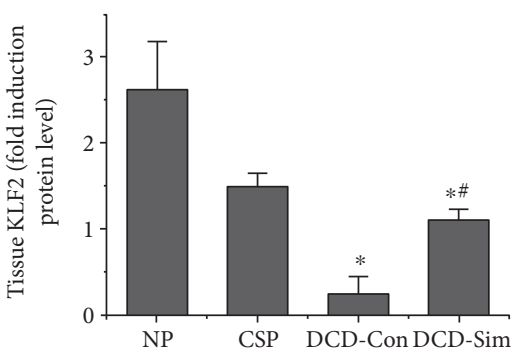

(b)

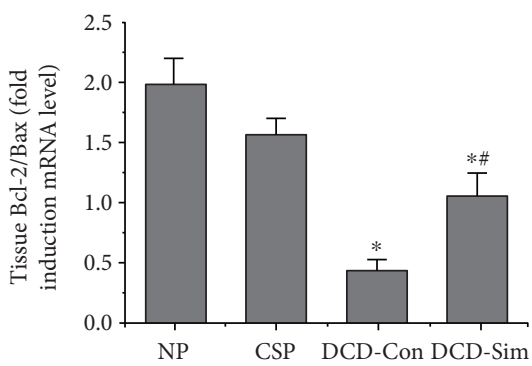

(d)

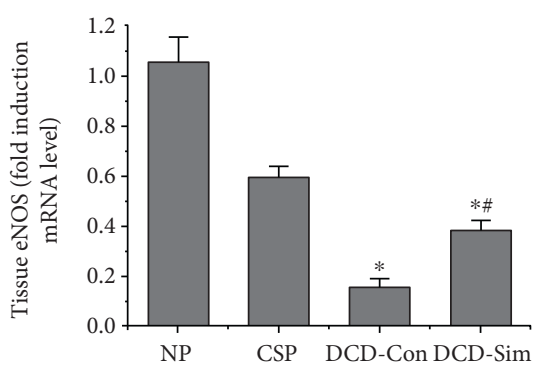

(f)

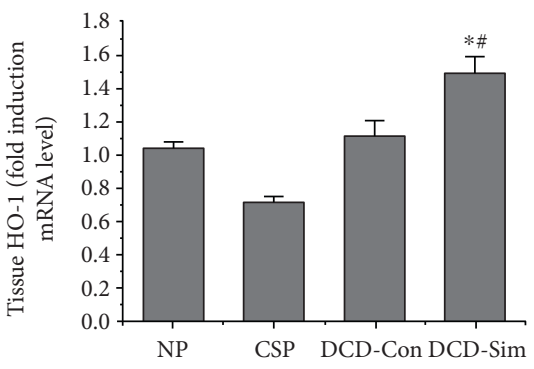

(h)

FIGURE 3: Simvastatin pretreatment maintains the expression of KLF2 and its protective target genes (a). The protein expression of KLF2 (b), phosphorylation eNOS (c), Bcl-2/Bax (d), and $\beta$-actin were determined by Western blotting and the gray values were calculated $(n=6$ per group; ${ }^{*} P<0.05$ versus NP group, ${ }^{\#} P<0.05$ versus DCD-Con group). The mRNA expression of KLF2 (e), eNOS (f), TM (g), and HO-1 (h) were assessed by RT-PCR. The experiments were repeated three times and the data are shown as mean \pm SD $\left(n=6\right.$; ${ }^{*} P<0.05$ versus NP group, ${ }^{\#} P<0.05$ versus DCD-Con group).

in vivo. Unfortunately, the therapeutic strategies to improve IRI in DCD livers are currently limited. Importantly, recent researches have demonstrated that donor simvastatin pretreatment can protect against hepatic IRI in livers from healthy, cirrhotic, and obese animals [15, 17, 22-25]. Therefore, the purpose of this study was to determine whether DCD donor simvastatin treatment ameliorates warm and cold ischemia-induced injury in liver allografts subjected to $24 \mathrm{~h}$ cold preservation. The major novel findings that emerged from our experiments are as follows: (1) Donor simvastatin pretreatment improves DCD liver quality after reperfusion in rats. (2) The protective effect of simvastatin is mediated via induction of KLF2, eNOS, TM, and HO-1 to improve hepatic microcirculation. (3) DCD liver simvastatin pretreatment can inhibit innate immune responses particularly via suppressing TLR4 and alleviating the release of HMGB1. (4) DCD liver simvastatin pretreatment reduces release of mitochondrial ROS to prevent oxidative stress. (5) DCD liver simvastatin pretreatment inhibits release of inflammatory cytokines. 


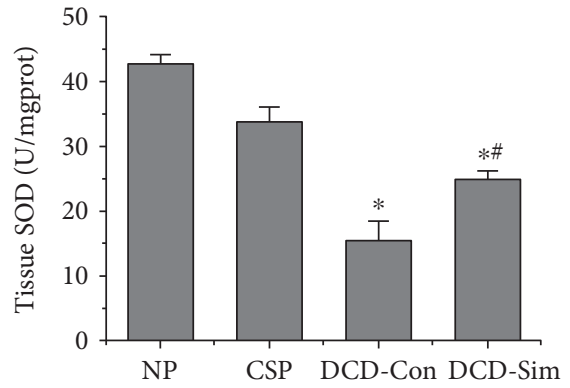

(a)

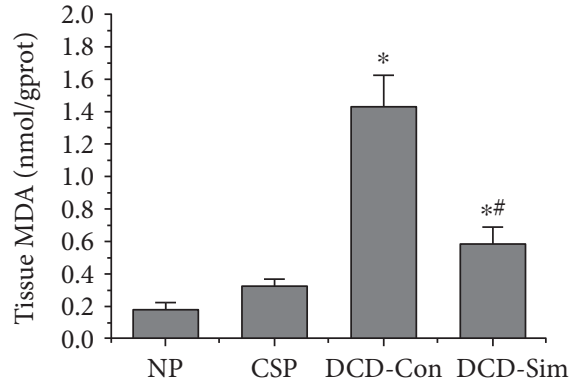

(b)

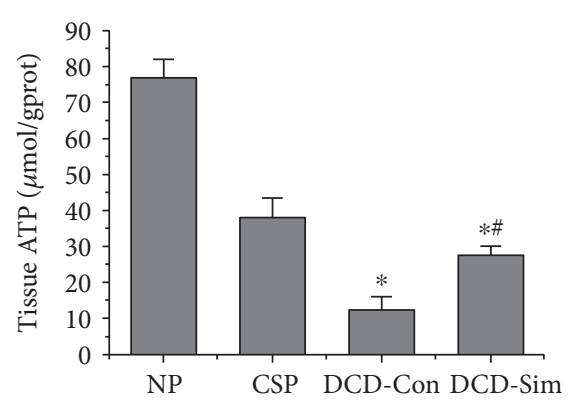

(c)

FIGURE 4: Simvastatin pretreatment reduces oxidative stress and improves ATP levels. (a) Liver tissue release of SOD level, (b) liver tissue release of MDA level, and (c) liver tissue release of ATP level $\left(n=6\right.$; $^{*} P<0.05$ versus NP group, ${ }^{\#} P<0.05$ versus DCD-Con group).

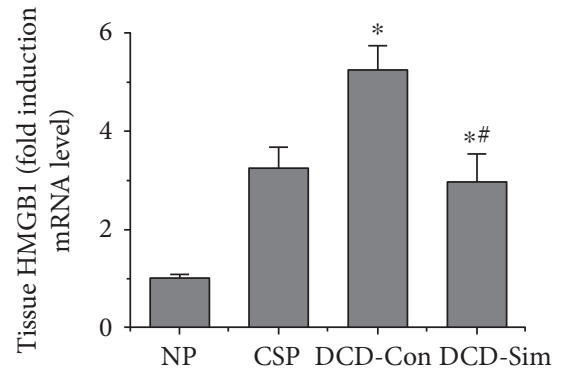

(a)

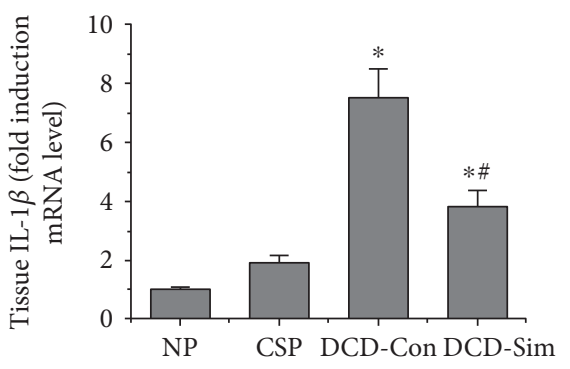

(d)

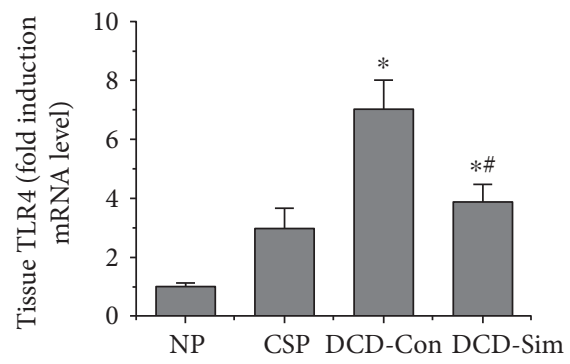

(b)

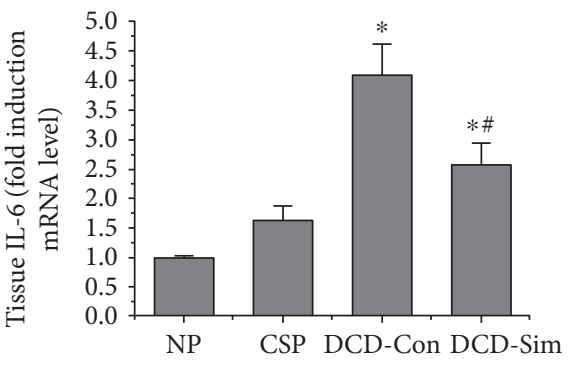

(e)

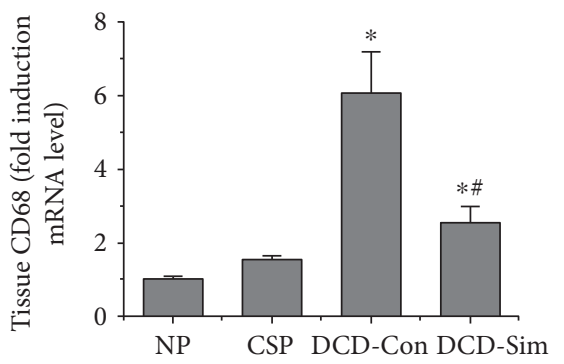

(c)

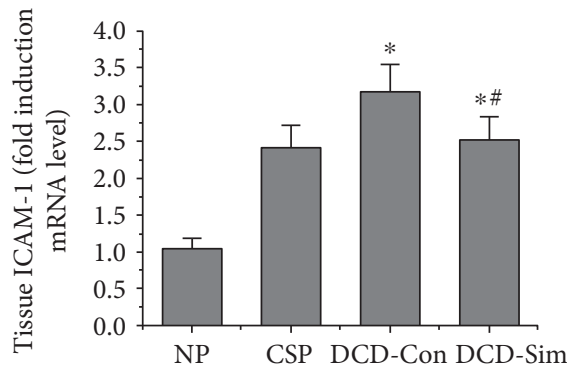

(f)

FIGURE 5: Simvastatin pretreatment inhibits innate immune responses and inflammatory. The mRNA expression of HMGB1 (a), TLR4 (b), CD68 (c), IL-1 $\beta$ (d), IL-6 (e), and ICAM-1(f) were assessed by RT-PCR. The experiments were repeated three times and the data are shown as mean $\pm \mathrm{SD}\left(n=6 ;{ }^{*} P<0.05\right.$ versus NP group, ${ }^{\#} P<0.05$ versus $\mathrm{DCD}$-Con group).

In the present study, we had well established the model of DCD livers in rats. Our results showed that DCD donor pretreatment with simvastatin significantly decreased perfusate levels of AST and ALT compared with those in the DCD control group at $1 \mathrm{~h}$ reperfusion subjected to $24 \mathrm{~h}$ cold preservation (Figures 1(a) and 1(b)). These results were consistent with the pathological changes (Figures 2(a) and 2(b)). Extensive hepatocyte necrosis, cytoplasmic vacuolization of hepatocytes, and neutrophil infiltration of liver tissues were observed in the DCD control group, which was obviously ameliorated in the simvastatin treatment group. In addition, bile production was markedly increased in the simvastatin treatment group compared to the DCD control group (Figure 1(c)). Our results demonstrated that simvastatin pretreatment attenuated perfusate liver enzyme levels and pathological changes and improved synthetic function in DCD livers after reperfusion.
The process of IRI in DCD livers are generally viewed as a serious event comprising the loss of oxygen supply and metabolic substrates during warm ischemia in vivo and preservation in vitro and the production of ROS and production of inflammatory mediators during reperfusion of blood flow. Moreover, during the process of organ hypoxia and reoxygenation, loss of blood pulsatile flow and vascular mechanical shear stress during allograft warm ischemia and preservation has been shown to play critical roles in IRI. Our results first demonstrated that DCD donor pretreatment with simvastatin had a better effect on functional recovery of DCD livers. However, the underlying mechanism of simvastatin in DCD liver IRI remains unclear. Indeed, recent studies have reported that flow cessation of the biomechanical stimulus by shear stress deteriorated the endothelial barrier homeostasis $[15,26]$ and activated proinflammatory [27] and prothrombotic pathways [27] by downregulating the expression of 

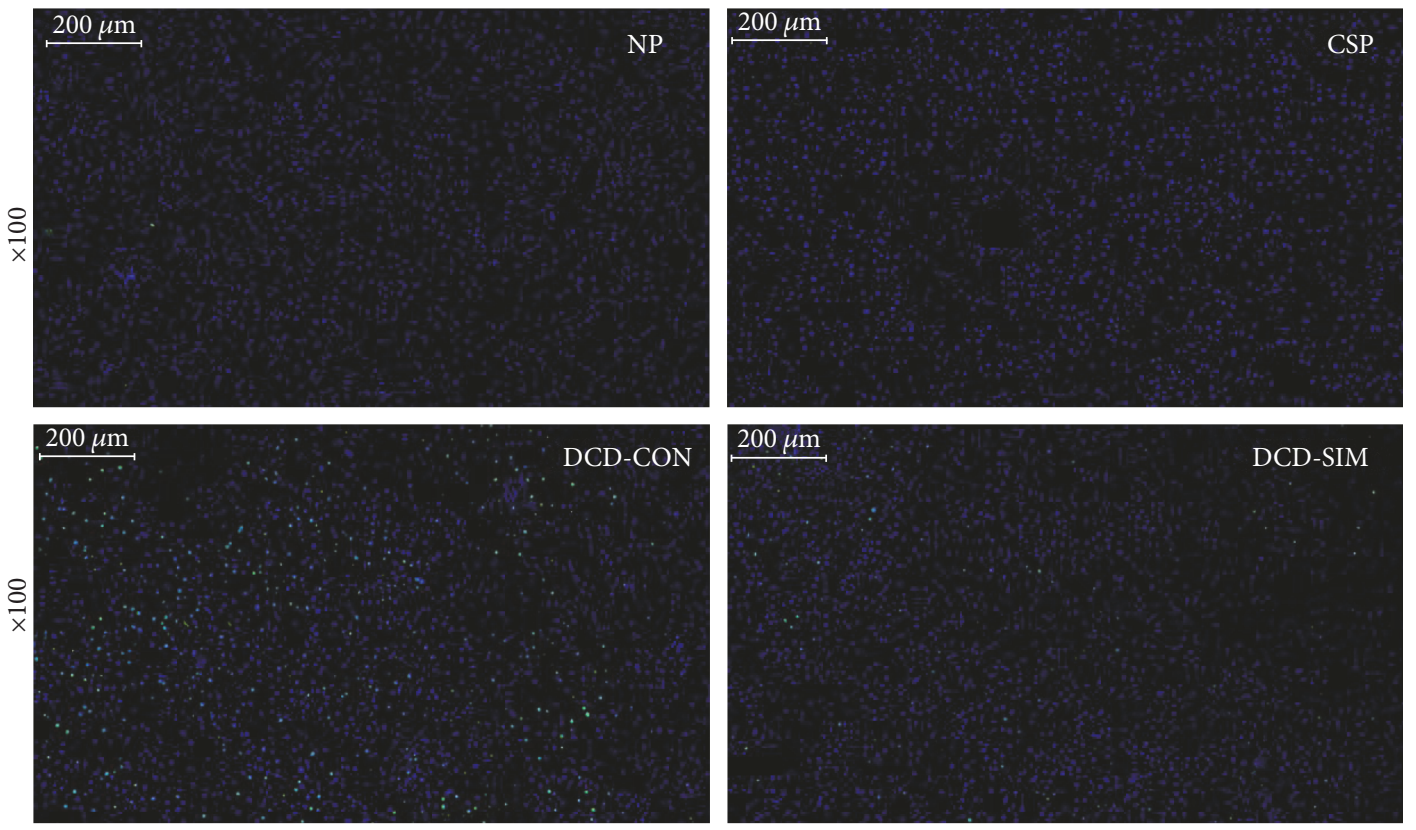

(a)

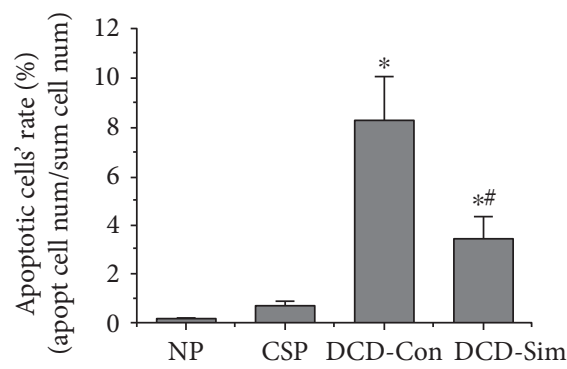

(b)

FIGURE 6: Simvastatin pretreatment suppresses hepatocyte apoptosis. (a) Representative images of TUNEL staining determined in livers from rats after $1 \mathrm{~h}$ warm reperfusion. Original magnification, 100x. (b) Quantitative analysis of liver apoptosis cells $\left(n=6\right.$; ${ }^{*} P<0.05$ versus NP group, ${ }^{\#} P<0.05$ versus DCD-Con group).

transcription factor KLF2. The present study showed that DCD livers after ex vivo reperfusion injury subjected to $24 \mathrm{~h}$ cold preservation downregulated the expression of KLF2 and p-eNOS (protein and mRNA levels), while DCD donor pretreatment with simvastatin upregulated the expression of KLF2 (protein and mRNA levels), eNOS (protein and mRNA levels), HO-1 (mRNA levels), and TM (mRNA levels) (Figures 3(a), 3(b), 3(c), 3(e), 3(f), and 3(g)). These observations demonstrated that the protective effects of simvastatin treatment in DCD donors may be due to the induction of the shear stress-regulated KLF2 and eNOS $[9,28]$.

Furthermore, several pathways have been demonstrated to be associated with KLF2, including eNOS pathway, HO1/ROS/Bcl-2 pathway, HMGB1/TLR4/NF-kappab pathway and inflammatory pathway. Hypoxia triggers the release of ROS and HMGB1 in different compartment molecules during procurement, preservation, and implantation [29, 30]. As the production of HMGB1 depends on the quantity of intracellular ROS, mitochondria was regarded as the source of the oxidative stress [30-33]. Therefore, it has recently been suggested that reduction of the initial release of ROS and HMGB1 molecule in donors could be important for subsequent prevention of immune and inflammatory responses [32, 34, 35]. In our study, we found that DCD donor pretreatment with simvastatin downregulated the mRNA expression of HMGB1 and TLR4 in the liver after $1 \mathrm{~h}$ reperfusion subjected to $24 \mathrm{~h}$ cold preservation compared to the DCD control group (Figures 5(a), 5(b), and 5(c)). In accordance with mitochondria function, the release of SOD increased and MDA levels of liver tissue lowered in DCD donor pretreatment with simvastatin group after reperfusion ex vivo compared to DCD control group (Figures 4(a) and 4(b)). This also might be attributed to the antioxidant properties of KLF2 through activation of nuclear factor erythroid-derived 2-like 2 (Nrf2) and upregulation expression of HO-1 [36, 37]. Next, the liver ATP was better functionally recovered in simvastatin group (Figure 4(c)). To explore the antiapoptotic or apoptotic mechanism of simvastatin on DCD livers, we evaluated the expression of $\mathrm{Bcl}-2$ and $\mathrm{Bax}$ protein in liver tissue. Our results showed that DCD livers after ex vivo reperfusion injury subjected to $24 \mathrm{~h}$ cold preservation upregulated Bax protein and downregulated $\mathrm{Bcl}-2$ protein while pretreatment with simvastatin significantly increased the expressions of 
Bcl-2 protein and downregulated the expression of Bax protein (Figures 3(a) and 3(c)). With TUNEL assay, DCD livers pretreated with simvastatin showed a significant lower apoptotic cell ratio, as compared with DCD control group (Figures 6(a) and 6(b)).

Finally, we investigated the expression of IL- $1 \beta$, IL- 6 , and ICAM-1 at the mRNA levels. Our results showed that DCD donor pretreatment with simvastatin significantly decreased the levels of these inflammatory cytokines compared with those in the DCD control group (Figures 5(e), 5(f), and $5(\mathrm{~g}))$, and thus the changes might also be depended on the anti-inflammatory potential of KLF2 [27, 38]. In turn, proinflammatory cytokines such as IL- $1 \beta$ also have been shown to repress KLF2 expression [39]. These observations demonstrated that the anti-inflammatory protective effects of DCD donor pretreatment with simvastatin reduced the neutrophil adhesion and extravasation.

Importantly, regarding the underlying mechanism on how KLF2 activators improve liver quality, Guixe et al. firstly suggested that simvastatin activated the upregulation of Rab7 and autophagic flux in the cold-stored liver sinusoidal endothelium [18]. Our results were consistent with the above findings and indicated the protective effects of simvastatin. It requires further research to explore the cross-talk between KLF2 and autophagy on DCD livers. Nevertheless, there were also some limitations in our study. Firstly, we used the ex vivo isolated liver reperfusion model to evaluate DCD liver quality but not the liver transplantation, which is closer to physiology. Next, the role of KLF2 in protection against DCD liver IRI could be further illustrated using genetic tools where KLF2 expression would be overexpressed or knocked down specifically.

In conclusion, the present study first demonstrates that DCD donor pretreatment with simvastatin improved DCD livers' functional recovery shortly after ex vivo reperfusion subjected to $24 \mathrm{~h}$ cold preservation and strongly protects liver cells. The protective mechanisms of simvastatinreduced DCD liver IRI might be mediated via upregulation of the flow-dependent protective genes KLF2 conferring anticoagulant, suppressing innate immune responses, antioxidant, antiapoptotic, and anti-inflammatory properties. Our results strongly supported pretreating potential DCD donors with simvastatin as a feasible strategy for liver organ protection. Ultimately, this possibility will require further clinical trials to evaluate the protective effects of statins in liver transplantation.

\section{Disclosure}

Zhongzhong Liu and Xingjian Zhang are co-first authors.

\section{Conflicts of Interest}

The authors declare that they have no conflict of interests.

\section{Authors' Contributions}

Zhongzhong Liu and Xingjian Zhang contributed equally to this work.

\section{Acknowledgments}

This study was supported by National Natural Science Foundation of China-Xinjiang joint fund (no. U1403222), National Natural Science Foundation of China (no. 81570079), Natural Science Foundation of Hubei Province (no. 2015CFA018), and The Fundamental Research Funds for the Central Universities (no. 2042016kf1002).

\section{References}

[1] P. Dutkowski, M. Linecker, M. L. DeOliveira, B. Müllhaupt, and P. A. Clavien, "Challenges to liver transplantation and strategies to improve outcomes," Gastroenterology, vol. 148, no. 2, pp. 307-323, 2015.

[2] R. W. Busuttil and K. Tanaka, "The utility of marginal donors in liver transplantation," Liver Transplantation, vol. 9, no. 7, pp. 651-663, 2003.

[3] A. E. Braat, J. J. Blok, H. Putter et al., "The eurotransplant donor risk index in liver transplantation: ET-DRI," American Journal of Transplantation, vol. 12, no. 10, pp. 2789-2796, 2012.

[4] J. C. Cutrn, M. G. Perrelli, B. Cavalieri, C. Peralta, J. Rosell Catafau, and G. Poli, "Microvascular dysfunction induced by reperfusion injury and protective effect of ischemic preconditioning," Free Radical Biology \& Medicine, vol. 33, no. 9, pp. 1200-1208, 2002.

[5] N. Selzner, H. Rudiger, R. Graf, and P. A. Clavien, "Protective strategies against ischemic injury of the liver," Gastroenterology, vol. 125, no. 3, pp. 917-936, 2003.

[6] G. B. Atkins and M. K. Jain, "Role of Kruppel-like transcription factors in endothelial biology," Circulation Research, vol. 100, no. 12, pp. 1686-1695, 2007.

[7] C. Collins and E. Tzima, "Hemodynamic forces in endothelial dysfunction and vascular aging," Experimental Gerontology, vol. 46, no. 2-3, pp. 185-188, 2011.

[8] L. Nayak, Z. Lin, and M. K. Jain, “"Go with the flow”: how Kruppel-like factor 2 regulates the vasoprotective effects of shear stress," Antioxidants \& Redox Signaling, vol. 15, no. 5, pp. 1449-1461, 2011.

[9] Z. Lin, A. Kumar, S. SenBanerjee et al., "Kruppel-like factor 2 (KLF2) regulates endothelial thrombotic function," Circulation Research, vol. 96, no. 5, pp. e48-e57, 2005.

[10] K. M. Parmar, H. B. Larman, G. Dai et al., "Integration of flow-dependent endothelial phenotypes by Kruppel-like factor 2," The Journal of Clinical Investigation, vol. 116, no. 1, pp. 49-58, 2006.

[11] A. Gnasso, C. Carallo, C. Irace et al., "Association between wall shear stress and flow-mediated vasodilation in healthy men," Atherosclerosis, vol. 156, no. 1, pp. 171-176, 2001.

[12] A. H. Wagner, T. Kohler, U. Ruckschloss, I. Just, and M. Hecker, "Improvement of nitric oxide-dependent vasodilatation by HMG-CoA reductase inhibitors through attenuation of endothelial superoxide anion formation," Arteriosclerosis, Thrombosis, and Vascular Biology, vol. 20, no. 1, pp. 61-69, 2000.

[13] T. S. Lee, C. C. Chang, Y. Zhu, and J. Y. Shyy, "Simvastatin induces heme oxygenase-1: a novel mechanism of vessel protection," Circulation, vol. 110, no. 10, pp. 12961302, 2004. 
[14] M. K. Jain and P. M. Ridker, "Anti-inflammatory effects of statins: clinical evidence and basic mechanisms," Nature Reviews Drug Discovery, vol. 4, no. 12, pp. 977-987, 2005.

[15] L. Russo, J. Gracia-Sancho, H. Garcia-Caldero et al., "Addition of simvastatin to cold storage solution prevents endothelial dysfunction in explanted rat livers," Hepatology, vol. 55, no. 3, pp. 921-930, 2012.

[16] J. Gracia-Sancho, H. Garcia-Caldero, D. Hide et al., "Simvastatin maintains function and viability of steatotic rat livers procured for transplantation," Journal of Hepatology, vol. 58, no. 6, pp. 1140-1146, 2013.

[17] D. Hide, M. Ortega-Ribera, J. C. Garcia-Pagan, C. Peralta, J. Bosch, and J. Gracia-Sancho, "Effects of warm ischemia and reperfusion on the liver microcirculatory phenotype of rats: underlying mechanisms and pharmacological therapy," Scientific Reports, vol. 6, no. 1, article 22107, 2016.

[18] S. Guixe-Muntet, F. C. de Mesquita, S. Vila et al., "Cross-talk between autophagy and KLF2 determines endothelial cell phenotype and microvascular function in acute liver injury," Journal of Hepatology, vol. 66, no. 1, pp. 86-94, 2017.

[19] A. Schlegel, R. Graf, P. A. Clavien, and P. Dutkowski, "Hypothermic oxygenated perfusion (HOPE) protects from biliary injury in a rodent model of DCD liver transplantation," Journal of Hepatology, vol. 59, no. 5, pp. 984-991, 2013.

[20] Z. Liu, Z. Zhong, J. Lan et al., "Mechanisms of hypothermic machine perfusion to decrease donation after cardiac death graft inflammation: through the pathway of upregulating expression of KLF2 and inhibiting TGF- $\beta$ signaling," Artificial Organs, vol. 41, no. 1, pp. 82-88, 2017.

[21] S. Suzuki, L. H. Toledo-Pereyra, F. J. Rodriguez, and D. Cejalvo, "Neutrophil infiltration as an important factor in liver ischemia and reperfusion injury. Modulating effects of FK506 and cyclosporine," Transplantation, vol. 55, no. 6, pp. 1265-1272, 1993.

[22] J. Trebicka, M. Hennenberg, W. Laleman et al., "Atorvastatin lowers portal pressure in cirrhotic rats by inhibition of RhoA/Rho-kinase and activation of endothelial nitric oxide synthase," Hepatology, vol. 46, no. 1, pp. 242-253, 2007.

[23] I. R. Lai, K. J. Chang, H. W. Tsai, and C. F. Chen, "Pharmacological preconditioning with simvastatin protects liver from ischemia-reperfusion injury by heme oxygenase-1 induction," Transplantation, vol. 85, no. 5, pp. 732-738, 2008.

[24] L. Llacuna, A. Fernandez, C. V. Montfort et al., "Targeting cholesterol at different levels in the mevalonate pathway protects fatty liver against ischemia-reperfusion injury," Journal of Hepatology, vol. 54, no. 5, pp. 1002-1010, 2011.

[25] H. Ajamieh, G. Farrell, H. J. Wong et al., "Atorvastatin protects obese mice against hepatic ischemia-reperfusion injury by Toll-like receptor-4 suppression and endothelial nitric oxide synthase activation," Journal of Gastroenterology and Hepatology, vol. 27, no. 8, pp. 1353-1361, 2012.

[26] J. Gracia-Sancho, G. Jr Villarreal, Y. Zhang et al., "Flow cessation triggers endothelial dysfunction during organ cold storage conditions: strategies for pharmacologic intervention," Transplantation, vol. 90, no. 2, pp. 142-149, 2010.

[27] S. SenBanerjee, Z. Lin, G. B. Atkins et al., "KLF2 is a novel transcriptional regulator of endothelial proinflammatory activation," The Journal of Experimental Medicine, vol. 199, no. 10, pp. 1305-1315, 2004.
[28] S. Sen-Banerjee, S. Mir, Z. Lin et al., "Kruppel-like factor 2 as a novel mediator of statin effects in endothelial cells," Circulation, vol. 112, no. 5, pp. 720-726, 2005.

[29] D. Tang, R. Kang, H. J. Zeh 3rd, and M. T. Lotze, "High-mobility group box 1, oxidative stress, and disease," Antioxidants \& Redox Signaling, vol. 14, no. 7, pp. 1315-1335, 2011.

[30] W. G. Land, "Emerging role of innate immunity in organ transplantation part II: potential of damage-associated molecular patterns to generate immunostimulatory dendritic cells," Transplantation Reviews, vol. 26, no. 2, pp. 73-87, 2012.

[31] R. F. van Golen, T. M. van Gulik, and M. Heger, "Mechanistic overview of reactive species-induced degradation of the endothelial glycocalyx during hepatic ischemia/reperfusion injury," Free Radical Biology \& Medicine, vol. 52, no. 8, pp. 1382-1402, 2012.

[32] R. F. van Golen, T. M. van Gulik, and M. Heger, "The sterile immune response during hepatic ischemia/reperfusion," Cytokine \& Growth Factor Reviews, vol. 23, no. 3, pp. 6984, 2012.

[33] C. Peralta, M. B. Jimenez-Castro, and J. Gracia-Sancho, "Hepatic ischemia and reperfusion injury: effects on the liver sinusoidal milieu," Journal of Hepatology, vol. 59, no. 5, pp. 1094-1106, 2013.

[34] A. Schlegel, P. Kron, R. Graf, P. Dutkowski, and P. A. Clavien, "Warm vs. cold perfusion techniques to rescue rodent liver grafts," Journal of Hepatology, vol. 61, no. 6, pp. 1267-1275, 2014.

[35] A. Schlegel, P. Kron, R. Graf, P. A. Clavien, and P. Dutkowski, "Hypothermic oxygenated perfusion (HOPE) downregulates the immune response in a rat model of liver transplantation," Annals of Surgery, vol. 260, no. 5, pp. 931-938, 2014.

[36] J. O. Fledderus, R. A. Boon, O. L. Volger et al., "KLF2 primes the antioxidant transcription factor $\mathrm{Nrf} 2$ for activation in endothelial cells," Arteriosclerosis, Thrombosis, and Vascular Biology, vol. 28, no. 7, pp. 1339-1346, 2008.

[37] G. Marrone, R. Maeso-Diaz, G. Garcia-Cardena et al., "KLF2 exerts antifibrotic and vasoprotective effects in cirrhotic rat livers: behind the molecular mechanisms of statins," Gut, vol. 64, no. 9, pp. 1434-1443, 2015.

[38] D. X. Bu, M. Tarrio, N. Grabie et al., "Statin-induced Kruppellike factor 2 expression in human and mouse T cells reduces inflammatory and pathogenic responses," The Journal of Clinical Investigation, vol. 120, no. 6, pp. 1961-1970, 2010.

[39] A. Kumar, Z. Lin, S. SenBanerjee, and M. K. Jain, "Tumor necrosis factor alpha-mediated reduction of KLF2 is due to inhibition of MEF2 by NF-kB and histone deacetylases," Molecular and Cellular Biology, vol. 25, no. 14, pp. 58935903, 2005. 


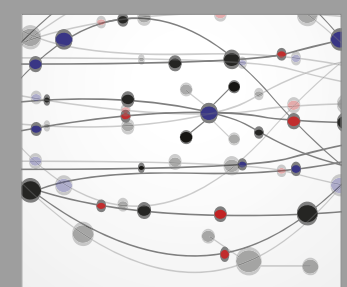

The Scientific World Journal
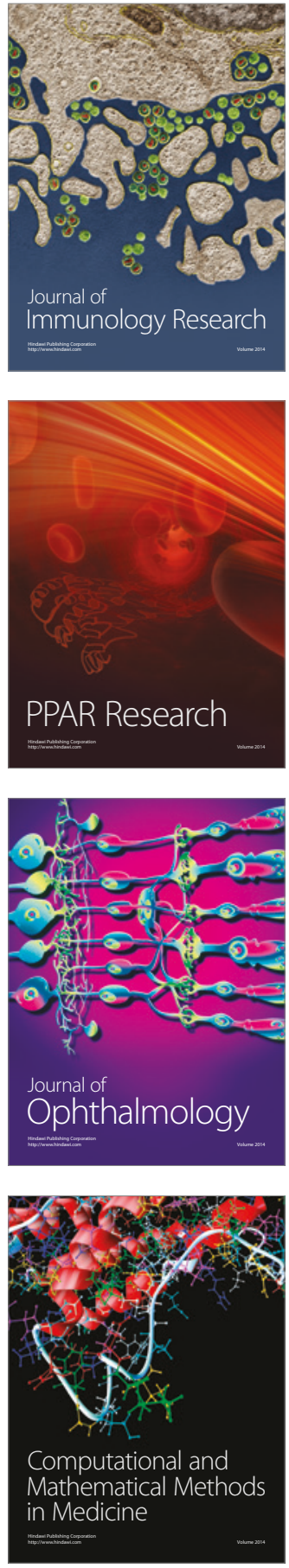

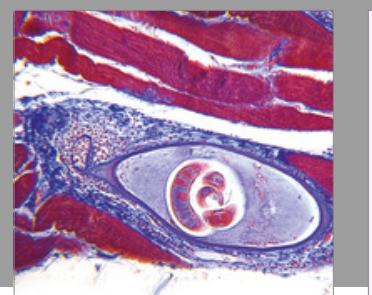

Gastroenterology Research and Practice
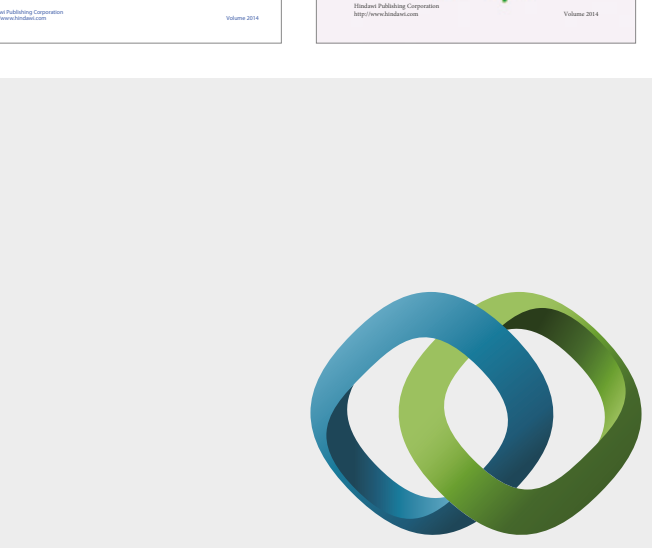

\section{Hindawi}

Submit your manuscripts at

https://www.hindawi.com
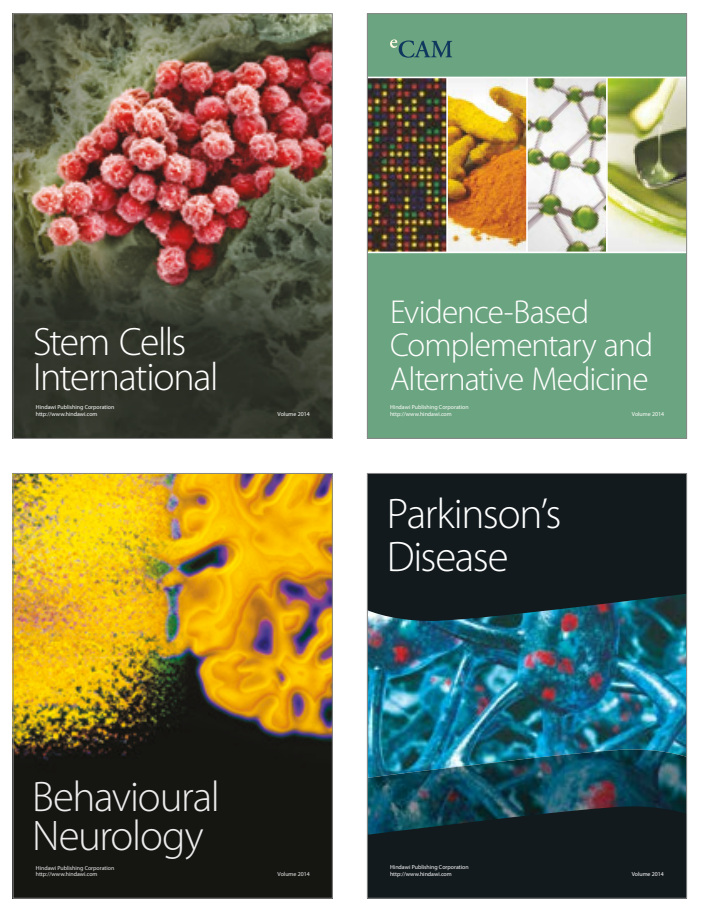
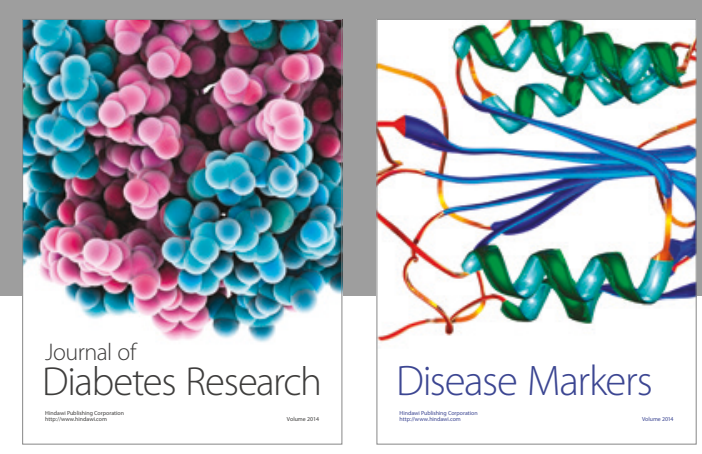

Disease Markers
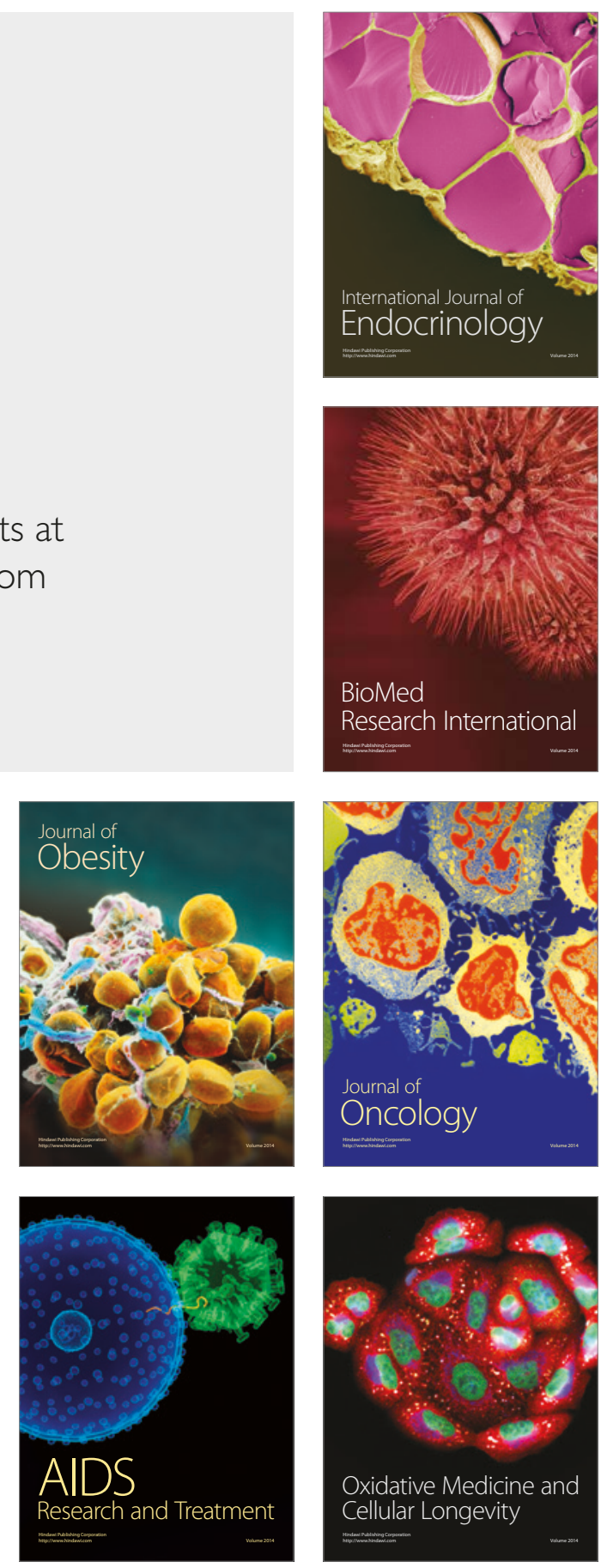